\title{
Image Analysis with Local Binary Patterns
}

\author{
Matti Pietikäinen \\ Machine Vision Group, \\ Infotech Oulu and Department of Electrical and Information Engineering, \\ P.O. Box 4500, FI-90014 University of Oulu, Finland \\ mkp@ee.oulu.fi, http://www.ee.oulu.fi/mvg
}

\begin{abstract}
The local binary pattern approach has evolved to represent a significant breakthrough in texture analysis, outperforming earlier methods in many applications. Perhaps the most important property of the LBP operator in real-world applications is its tolerance against illumination changes. Another equally important is its computational simplicity, which makes it possible to analyze images in challenging real-time settings. Recently, we have begun to study image analysis tasks which have not been generally considered texture analysis problems. Our excellent results suggest that texture and the ideas behind the LBP methodology could have a much wider role in image analysis and computer vision than was thought before.
\end{abstract}

\section{Introduction}

Image texture analysis is an important fundamental problem in computer vision. During the past few years, we have developed theoretically and computationally simple, but very efficient nonparametric methodology for texture analysis based on Local Binary Patterns (LBP). The LBP texture analysis operator is defined as a grayscale invariant texture measure, derived from a general definition of texture in a local neighborhood. For each pixel in an image, a binary code is produced by thresholding its value with the value of the center pixel. A histogram is created to collect up the occurrences of different binary patterns. The basic version of the LBP operator considers only the eight neighbors of a pixel, but the definition has been extended to include all circular neighborhoods with any number of pixels [1, 2, 3].

Through its extensions, the LBP operator has been made into a really powerful measure of image texture, showing excellent results in terms of accuracy and computational complexity in many empirical studies. The LBP operator can be seen as a unifying approach to the traditionally divergent statistical and structural models of texture analysis. Perhaps the most important property of the LBP operator in realworld applications is its tolerance against illumination changes. Another equally important is its computational simplicity, which makes it possible to analyze images in challenging real-time settings.

The LBP method has already been used in a large number of applications all over the world, including visual inspection, image retrieval, remote sensing, biomedical image analysis, face image analysis, motion analysis, environment modeling, and outdoor scene analysis. For a bibliography of LBP-related research, see [4]. 
Recently, we have begun to study machine vision tasks which have not been previously considered texture analysis problems. The LBP methodology has been adapted to outdoor scene classification, face recognition, face detection, facial expression recognition and content based image retrieval with excellent success. We have also developed the first texture-based method for subtracting the background and detecting moving objects in real time. A short description of this recent progress is presented in the following section.

\section{Recent Progress}

In [5], we proposed a new method for view-based recognition of 3D textured surfaces using multiple LBP histograms as texture models. Our method provided the leading performance for the Columbia-Utrect database (CUReT) textures taken in different views and illuminations. The method also performed well in pixel-based classification of outdoor scene textures. These images had wide variability within and between images due to changes in illumination, shadows, foreshortening, self-occlusion, and non-homogeneity of the texture classes.

Finding proper features and representative training samples can be very problematic in this kind of problems. Earlier we proposed a visualization based approach for training a texture classifier, in which LBP features are used for texture description and a self-organizing map (SOM) is employed for visual training and classification [6]. Recently, we developed a more comprehensive framework for texture image labeling [7]. Textures are modeled with complementary measures including various versions of the LBP and Gabor features. Combined use of active learning, co-training, and visualization based learning is applied to feature data, enabling comprehensive, accurate, and user friendly texture labeling.

A novel approach to face recognition was developed which considers both shape and texture information to represent face images [8]. The face area is first divided into several small regions from which the LBP features are extracted and concatenated into an enhanced feature vector to be used as a face descriptor. In extensive experiments using FERET test images and protocol, considering variations in facial expressions, lighting and aging of the subjects, our methodology outperformed the state-of-the-art methods. However, it was unclear whether the high performance was due to the use of local regions (instead of an holistic approach) or to the discriminative power of LBP. Experimental results with four different texture descriptors clearly showed the superiority of the LBP based approach [9].

A compact LBP based descriptor was also developed for face detection and for the recognition of low-resolution face images [10]. Considering the derived feature space, a second-degree polynomial kernel SVM classifier was trained to detect frontal faces in gray scale images. Experimental results using several complex images showed that our approach performs favorably compared to the state-of-the-art. Additionally, experiments with detecting and recognizing low-resolution faces from video sequences were carried out, demonstrating that the same facial representation can be efficiently used for both detection and recognition.

Two approaches to facial expression recognition from static images were developed using LBP histograms computed over non-overlapping blocks for face 
description. In the first method, the Linear Programming technique is adopted to classify seven facial expressions (anger, disgust, fear, happiness, sadness, surprise and neutral) [11]. In another approach, a coarse-to-fine classification scheme was used [12]. Good results were obtained for the Japanese Female Facial Expression (JAFFE) database used in the experiments.

Approaches to using LBP in content-based image retrieval were also studied [13]. Block based methods dividing the query and database images (or database images only) into blocks and comparing their LBP histograms were found to perform significantly better that methods based on global LBP histograms. The results for the block based LBP approach were also better than those obtained with the widely used color correlogram features. Image databases taken from the Corel Image Gallery and from a German stamp database were used in experiments.

A novel texture-based method for modeling the background and detecting moving objects from video sequences was developed $[14,15]$. Each pixel is modeled as a group of adaptive local binary pattern histograms that are calculated over a circular region around the pixel. The method was evaluated against several video sequences including both indoor and outdoor scenes. It was shown to be tolerant to illumination variations, the multimodality of the background, and the introduction or removal of background objects. Furthermore, the method is capable for real-time processing.

\section{Conclusions}

The local binary pattern approach has evolved to represent a significant breakthrough in texture analysis, outperforming earlier methods in many applications. Recently, we have begun to study image analysis tasks which have not been generally considered texture analysis problems. Our excellent results suggest that texture and the ideas behind the LBP methodology could have a much wider role in image analysis and computer vision than was thought before. Our future plan is to explore this thoroughly.

\section{Acknowledgments}

I wish to thank Timo Ahonen, Abdenour Hadid, Marko Heikkilä, Topi Mäenpää, Timo Ojala, Valtteri Takala, Markus Turtinen, and Feng Xiaoyi for their contributions. The financial support of the Academy of Finland is gratefully acknowledged.

\section{References}

1. Ojala, T., Pietikäinen, M., Harwood, D.: A Comparative Study of Texture Measures with Classification Based on Feature Distributions. Pattern Recognition 29 (1996) 51-59

2. Ojala, T., Pietikäinen, M., Mäenpää, T.: Multiresolution Gray-Scale and Rotation Invariant Texture Classification with Local Binary Patterns. IEEE Transactions on Pattern Analysis and Machine Intelligence 24 (2002) 971 - 987 
3. Mäenpää, T., Pietikäinen, M.: Texture Analysis with Local Binary Patterns. In: Chen, C.H., Wang, P.S.P. (eds.): Handbook of Pattern Recognition and Computer Vision, $3^{\text {rd }}$ edn. World Scientific (2005) 197-216

4. http://www.ee.oulu.fi/research/imag/texture/

5. Pietikäinen, M., Nurmela, T., Mäenpää, T., Turtinen, M.: View-Based Recognition of Real-World Textures. Pattern Recognition 37 (2004) 313-323

6. Turtinen, M., Pietikäinen, M.: Visual Training and Classification of Textured Scene Images. In: The 3rd International Workshop on Texture Analysis and Synthesis (2003) 101-106

7. Turtinen, M., Pietikäinen, M.: Labeling of Textured Data with Co-Training and Active Learning. In review.

8. Ahonen, T., Hadid, A., Pietikäinen, M.: Face Recognition with Local Binary Patterns. In: Computer Vision, ECCV 2004 Proceedings, Lecture Notes in Computer Science 3021 (2004) 469-481

9. Ahonen, T., Pietikäinen, M., Hadid, A., Mäenpää, T.: Face Recognition Based on the Appearance of Local Regions. In: 17th International Conference on Pattern Recognition (2004) III: $153-156$

10. Hadid, A., Pietikäinen, M., Ahonen, T.: A Discriminative Feature Space for Detecting and Recognizing Faces. In: IEEE Conference on Computer Vision and Pattern Recognition (2004) II: 797-804

11. Feng, X., Pietikäinen, M., Hadid, A.: Facial Expression Recognition with Local Binary Patterns and Linear Programming. Pattern Recognition and Image Analysis 15 (2005) 550552

12. Feng, X., Hadid, A., Pietikäinen, M.: A Coarse-to-Fine Classification Scheme for Facial Expression Recognition. In: Image Analysis and Recognition, ICIAR 2004 Proceedings, Lecture Notes in Computer Science 3212 (2004) II: 668-675

13. Takala, V., Ahonen, T., Pietikäinen, M.: Block-Based Methods for Image Retrieval Using Local Binary Patterns. In: Image Analysis, SCIA 2005 Proceedings, Lecture Notes in Computer Science 3540 (2005)

14. Heikkilä, M., Pietikäinen, M., Heikkilä, J.: A Texture-Based Method for Detecting Moving Objects. In: The 15th British Machine Vision Conference (2004) I: 187-196

15. Heikkilä, M., Pietikäinen, M.: A Texture-Based Method for Modeling the Background and Detecting Moving Objects. In review. 